\title{
Genotypic and Phenotypic Correlations between Leaf-Rust Disease and Leaf Morphology and its Ratio in Arabica Coffee
}

\author{
(Korelasi Genotipik dan Fenotipik antara Penyakit Karat Daun dan \\ Morfologi Daun dan Rasionya pada Kopi Arabica)
}

\author{
Sabam Malau \\ (Diterima Juli 2019/Disetujui Mei 2020)
}

\begin{abstract}
Selection parameter in coffee breeding for leaf rust (Hemeleia vastatrix) resistance is very important. Breeders used leaf-rust severity and leaf-rust incidence as parameters of direct selection. However, scientific proof is not yet available whether leaf morphology can be used as a parameter of indirect selection. The objective of this research was to seek the possibility of leaf morphology parameter and its ratio to be used as selection criteria through analyses of genotypic and phenotypic correlations of parameter of rust disease and parameter of leaf morphology and its ratio. The result revealed that genotypes showed significant variations in leaf-rust severity (5.21-25.84\%), leaf morphology, and leaf-morphology ratio. Leaf length to leaf width ratio, leaf length to leaf area ratio, and leaf width to leaf weight ratio were not affected by the environment. Leaf-rust severity performed highly significant positive genotypic and phenotypic correlations the ratio of with leaf length to leaf area. For selection criteria, leaf-rust severity could be better used rather than leaf-rust incidence and branch-rust incidence. The ratio of leaf length to leaf area could also be used as an indirect selection criterion because the ratio showed a highly significant genotypic correlation with

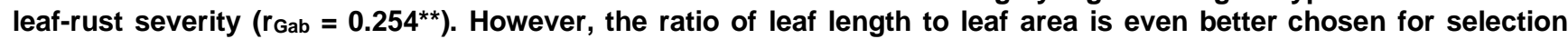
criteria rather than leaf-rust severity because the ratio was not affected by the environment.
\end{abstract}

Keywords: fungus, Hemeleia vastatrix, indirect selection

\section{ABSTRAK}

Parameter seleksi dalam pemuliaan kopi untuk ketahanan karat daun (Hemeleia vastatrix) sangat penting. Pemulia menggunakan keparahan karat daun dan insiden karat daun sebagai parameter seleksi langsung. Akan tetapi, bukti ilmiah belum tersedia apakah morfologi daun dapat digunakan sebagai parameter seleksi secara tidak langsung. Tujuan penelitian ini adalah untuk mencari kemungkinan apakah parameter morfologi daun dan rasionya dapat digunakan sebagai kriteria seleksi melalui analisis korelasi genotipik dan fenotipik parameter penyakit karat dan parameter morfologi daun dan rasionya. Hasil penelitian menunjukkan bahwa genotipe menunjukkan variasi yang signifikan dalam tingkat keparahan karat daun (5,21-25,84\%), morfologi daun, dan rasio morfologi daun. Rasio antara panjang daun dan lebar daun, rasio antara panjang daun dan luas daun, dan rasio antara lebar daun dan bobot daun tidak dipengaruhi oleh lingkungan. Keparahan karat daun menunjukkan korelasi genotipik dan fenotipik yang positif signifikan dan tinggi dengan rasio antara panjang daun dan luas daun. Untuk kriteria seleksi, tingkat keparahan karat daun lebih baik digunakan daripada insiden karat daun dan insiden karat cabang. Rasio antara panjang daun dan luas daun juga dapat digunakan sebagai kriteria seleksi tidak langsung karena rasio tersebut menunjukkan korelasi genotipe yang sangat signifikan dengan tingkat keparahan karat daun ( $\left.\mathrm{r}_{\mathrm{Gab}}=0,254^{\star \star}\right)$. Akan tetapi, rasio antara panjang daun dan luas daun lebih baik dipilih untuk kriteria seleksi daripada keparahan karat daun karena rasio itu tidak dipengaruhi oleh lingkungan.

Kata kunci: Hemeleia vastatrix, jamur, seleksi tidak langsung

\section{INTRODUCTION}

Coffee is very important for Indonesia to generate income and jobs. In 2016, Indonesia produced around 190 thousand tons of Arabica coffee and 474 thousand tons of Robusta coffee (DGEC 2017). This country has 1.25 million ha of coffee farms and 1.78 million of coffee farmers (households). As one of the main

Agroecotechnology Department, Agriculture Faculty, Universitas HKBP Nommensen, Jalan Sutomo 4-A, Medan 20234

Coresponden Author: Email: sabam.malau@uhn.ac.id production area of Arabica coffee in Indonesia, North Sumatra Province produced 53.2 thousand tons of green beans on a total of 63.3 thousand ha of coffee growing area which was income source for 110.6 thousand farmers (households).

Fungus Hemileia vastatrix cause rust disease on leaf of coffee plant. The fungus lives in the underside of the leaf. Fungi that live on the bottom surface of the leaves (Figure 1) can cover up to $100 \%$ of the surface. The leaves are then damaged (Figure 2) and eventually fall off.

This fungus severely destructed coffee plants in many countries in Asia, Central America, and Africa 


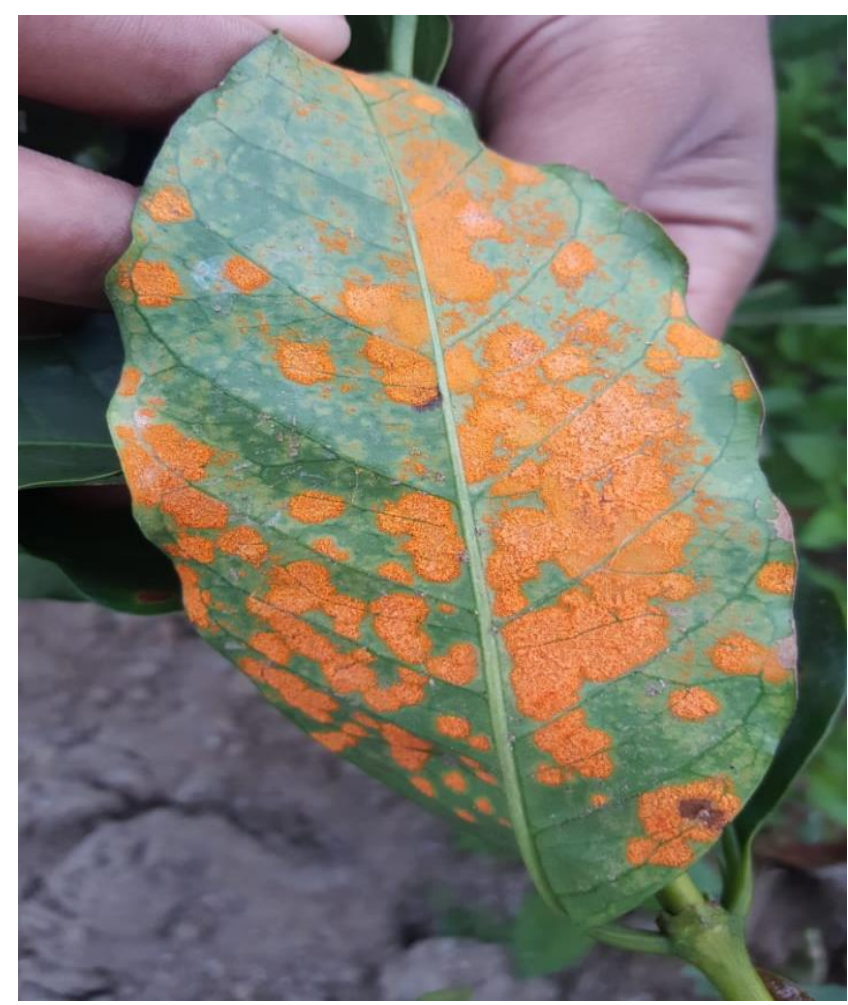

Figure 1 Fungi Hemileia vastatrix live on the bottom surface of the leaf.

(McCook 2006; Zeru et al. 2009; Jeffuka et al. 2010; Avelino et al. 2015; Talhinhas et al. 2017). In North Sumatra Province of Indonesia, this fungus covered some farms with leaf-rust severity from $1 \%$ to $45 \%$ (average 15.8\%) (Siska et al. 2018).

Resistant genotypes of coffee could be found through crossbreeding (Jeffuka et al. 2010; Sera et al. (2010); Hindorf \& Omondi 2011; Rodrigues et al. 2012; Caicedo et al. 2013, Andreazi et al. 2015) or in existing genotypes having genotypic variation and phenotypic variation (Carvalho et al. 2011; Harni et al. 2015). In existing genotypes of Arabica coffee in North Sumatera Province, genotypes are rich in variabilities in many important traits such as rust resistance (Malau 2019a; Malau et al. 2019a), morphological traits (Malau \& Pandiangan 2018), seed-germination ability (Malau et al. 2018a), water-stress tolerance (Malau et al. 2018b), adaptability to climate changing (Malau et al. 2018c), and taste (Malau et al. 2017; Malau et al. 2018d; Malau et al. 2019b; Malau 2019b).

So far, leaf-rust severity and leaf-rust incidence were used by coffee breeder as direct selection parameters. It seems to be important to study the correlation between rust disease with leaf morphology because spores of this fungus only live on coffee leaf, and infect through the stomata on the underside of the leaf (López-Bravo et al. 2012; Boudrot et al. 2016). The objective of this research was to genotypically and phenotypically correlate parameter of rust disease with leaf morphology and the ratios of leaf-morphology parameters. It was hypothesized that rust disease had significant genotypic and phenotypic correlations with

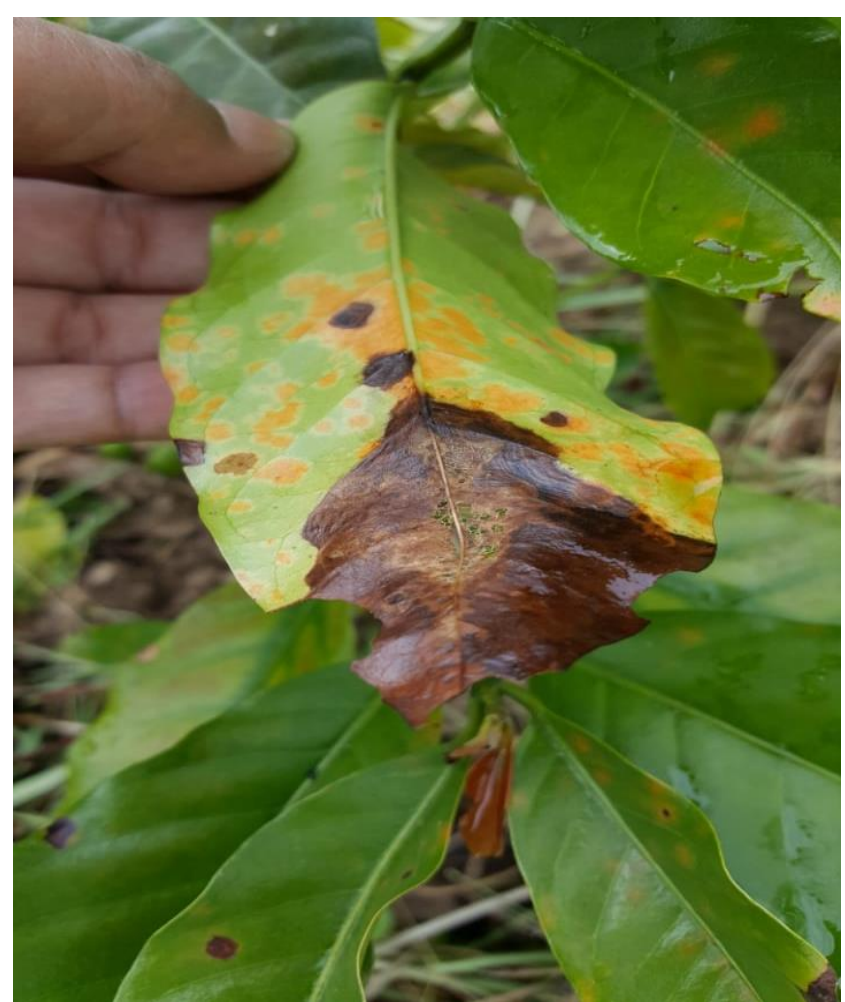

Figure 2 The leaf is damaged by fungi Hemileia vastatrix and eventually falls off.

leaf morphology and its ratios. The result of this research was expected to contribute to a method of coffee breeding for rust resistance.

\section{MATERIAL AND METHODS}

The research was conducted in Regencies of Dairi, Pakpak Bharat, Simalungun, Samosir, Humbang Hasundutan, Tapanuli Utara, and Toba Samosir of North Sumatra Province in June 2017. According to Sudrajat (2009), the climate zones of the research locations were A1, B1, C1, D1, D2, E1, and E2 for Humbang Hasundutan, Simalungun, Pakpak Bharat, Samosir, Dairi, North Tapanuli, and Toba Samosir Regencies, respectively (Table 1 ). The length of rainy seasons and rainfall was different enough. Wet season and dry season have rainfall in the amount of more than $200 \mathrm{~mm}$ and less than $100 \mathrm{~mm}$ per month, respectively. In the period of 1970-2017, the average range of temperature was $21.8-23.6^{\circ} \mathrm{C}$ (BMKG 2017).

The nested design with three factors (regency, sub regency, and genotype) was used (Quinn \& Keough, 2002). In each regency, two sub regencies nested in regency were selected. In each sub regency, six farms nested in sub regency were chosen. Therefore, $7 \times 2 \times$ $6=84$ farms were selected. The farms were treated as genotypes. Ten plants with age 6-7 years of each farms were selected randomly which were treated as genotype. Leaf length $(L)$ and leaf width (W) were measured while estimated leaf area (ELA) was calculated using formula ELA $=0.99927^{\star}\left(\mathrm{L}^{*}(-0.14757\right.$ 
Table 1 Length of rainy season, length of dry season, minimum rainfall, maximum rainfall, average rainfall, and temperature of climate zones

\begin{tabular}{lccccccc}
\hline & & & & Regency & & North \\
\cline { 2 - 7 } & $\begin{array}{c}\text { Humbang } \\
\text { Hasundutan }\end{array}$ & Simalungun & $\begin{array}{c}\text { Pakpak } \\
\text { Bharat }\end{array}$ & Samosir & Dairi & $\begin{array}{c}\text { Toba } \\
\text { Tapanuli }\end{array}$ & Samosir \\
\hline CZ & A1 & B1 & C1 & D1 & D2 & E1 & E2 \\
LoRS & 0 & 0 & 1 & 1 & 2 & 1 & 2 \\
MinR & 3108 & 2595 & 1750 & 1705 & 1749 & 1615 & 1172 \\
MaxR & 4388 & 3104 & 3957 & 3085 & 2409 & 2145 & 2233 \\
AvR & 3822 & 2933 & 2729 & 2274 & 1911 & 1922 & 1685 \\
AvT & 23.3 & 23.1 & 22.9 & 22.5 & 21.8 & 23.6 & 23.6 \\
\hline
\end{tabular}

Source: Sudrajat (2009); BMKG (2017).

Description: $\quad C Z=$ Climate zone, LoRS $=$ Length of rainy season (months), MinR $=$ minimum rainfall $(\mathrm{mm}$ per year), MaxR $=$ maximum rainfall ( $\mathrm{mm}$ per year), $\mathrm{AvR}=$ average rainfall ( $\mathrm{mm}$ per year), and $\mathrm{AvT}=$ average temperature $\left({ }^{\circ} \mathrm{C}\right)$.

+ 0.60986*W)) (Unigarro-Muñoz et al. 2015). Branchrust incidence (BRI), leaf-rust incidence (LRI), and leafrust severity (LRS) were calculated. The proportion of rust-infected branches from total branches (\%) is branch-rust incidence. A branch with at least one leaf with powdery lesion orange-yellow color due to sporulation on the underside of leaf was defined as a rust-infected branch. All branches were checked. BRI of a plant $\left(B R I_{P}\right)$ was calculated with the formula:

$$
\mathrm{BRI}_{\mathrm{p}}=\frac{\text { number of rust infected branches }}{\text { total branches }} \times 100 \% \text {. }
$$

Averaged BRI per plant of a genotype in a farm $\left(B R I_{g}\right)$ was calculated with the formula:

$$
\mathrm{BRI}_{\mathrm{g}}=\frac{\sum_{\mathrm{p}=1}^{\mathrm{q}} \mathrm{BRI}_{\mathrm{p}}}{\mathrm{q}}
$$

Whereby:

$\begin{array}{ll}\mathrm{BRI} & =\text { BRI per plant of } \mathrm{p}^{\text {th }} \text { plant }(\mathrm{p}=1,2, \ldots \mathrm{q}) \\ \mathrm{q} & =\text { number of plants }\end{array}$

The proportion of rust-infected leaves from total leaves (\%) of a rust-infected branch is leaf-rust incidence (LRI). A leaf with powdery lesion orangeyellow color due to sporulation on the underside of leaf was treated as a rust-infected leaf. To determine LRI per plant, one rust-infected branch from the lowest part of plant, one rust-infected branch from the middle part of plant, and one rust-infected branch from the most upper part of plant were selected. LRI of all sample plants were checked. LRI of a rust-infected branch

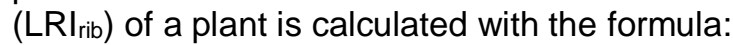

$$
\begin{gathered}
\mathrm{LRI}_{\mathrm{ribp}}= \\
\frac{\text { number of rust }- \text { infected leaves of the selected branches rusted }}{\text { total leaves of the selected branches rusted }} \times 100 \%
\end{gathered}
$$

Averaged $\left.L R\right|_{\text {rib }}$ per rust-infected branch of a genotype in an experimental plot ( $\left(\mathrm{LR} \mathrm{I}_{\text {ribg }}\right)$ was counted with the formula:

$$
\mathrm{LRI}_{\text {ribg }}=\frac{\sum_{\mathrm{p}=1}^{\mathrm{q}} \mathrm{LRI}_{\text {ribp }}}{\mathrm{q}}
$$

Whereby:

$$
\begin{array}{ll}
L R I_{\text {ribp }} & =L R I_{\text {rib }} \text { of } p^{\text {th }} \text { plant }(p=1,2, \ldots q) \\
q & =\text { Number of plants }
\end{array}
$$

The proportion of leaf-area rusted (\%) was defined as leaf-rust severity (LRS). To calculate LRS, all rustinfected leaves used in determining the LRI were used. Assessment of LRS was determined using a diagrammatic scale (Jeffuka et al 2010). Leaf-rust severity (LRS rilp) of a plant was determined with the formula:

$$
L R S_{\text {rilp }}=\frac{\sum_{w=1}^{z} L R S_{w}}{Z} \times 100 \%
$$

Whereby:

$\mathrm{LRS}_{\mathrm{w}}=$ LRS of $\mathrm{w}^{\text {th }}$ leaf $(\mathrm{w}=1,2, \ldots . \mathrm{z})$

$\mathrm{z} \quad=$ Number of rust infected leaves

Averaged LRS ril of a genotype in a farm (LRS rilg) was counted with the formula:

$$
\mathrm{LRS}_{\text {rilg }}=\frac{\sum_{\mathrm{p}=1}^{\mathrm{q}} \mathrm{LRS}_{\text {rilp }}}{\mathrm{q}}
$$

Whereby:

$$
\begin{array}{ll}
\text { LRS }_{\text {rilp }} & =\text { LRS }_{\text {ril }} \text { of } p^{\text {th }} \text { plant }(p=1,2, \ldots . q) \\
q & =\text { Number of plants }
\end{array}
$$

Genotypic and phenotypic correlation coefficients were then calculated. The first step was to create a dummy phenotype $(=a+b)$. Therefore, $\sigma^{2} a b=\sigma^{2} a+\sigma^{2} b$ $+2 \operatorname{cov}_{a b}$ where $\sigma^{2} a b=$ variance of the dummy phenotype, $\sigma_{a}^{2}=$ variance of phenotype $a, \sigma^{2} b=$ variance of phenotype $b$ and $\operatorname{cov}_{a b}=$ covariance of the dummy phenotype. The second step was to analyze the variance of the dummy phenotype by using the nested design method, then $\operatorname{cov}_{\mathrm{Gab}}{ }^{\prime} \mathrm{COV}_{\mathrm{Pab}} \sigma^{2} \mathrm{Gab}^{\prime} \sigma^{2} \mathrm{Pab}^{\prime}$ $\mathrm{r}_{\mathrm{Gab}}$ and $\mathrm{r}_{\mathrm{Pab}}$ could be calculated. Genetic correlation coefficient $r_{G a b}$ between two phenotypes ( $a$ and $b$ ) was calculated as $\mathrm{r}_{\mathrm{Gab}}=\operatorname{cov} \mathrm{Cab}_{\mathrm{Gab}} /\left(\sigma_{\mathrm{G}}^{2} \times \sigma^{2} \mathrm{~Gb}\right)^{0.5}$ and phenotypic correlation coefficient $\mathrm{r}_{\mathrm{Pab}}$ between two phenotypes was calculate as $\mathrm{r}_{\mathrm{Pab}}=\operatorname{cov}_{\mathrm{pab}} /\left(\sigma^{2} \mathrm{~Pa} \times \sigma^{2} \mathrm{~Pb}\right)^{0.5}$ where $\operatorname{cov}_{G a b}$ was genetic covariance between phenotypes $a$ and $b$, and covpab was phenotypic covariance between phenotypes $a$ and $b$ (Mayo 1987). The significant magnitude of $\mathrm{r}_{\mathrm{Gab}}$ and $\mathrm{r}_{\mathrm{Pab}}$ were tested by using critical $r$ tabular value at $\alpha=0.05$ and $\alpha=0.01$ using the degree of freedom of the error (Quinn \& Keough 2002). 


\section{RESULT AND DISCUSSION}

The regencies and sub regencies were significantly different in rust disease as well as in some leaf morphologies (Table 2). Leaf morphology is generally significantly affected by regency or sub regency or both. This indicates that environmental factors for growing coffee in general significantly affected the morphology of the leaves. Environmental components that influence the growth and development of coffee are climate zones, length of dry seasons, high elevation, temperature levels, and soil pH levels (Malau et al. 2018c).

Genotypes were highly significantly different in leafrust severity, leaf-rust incidence, branch-rust incidence, leaf length, leaf width, leaf weight, leaf area, and the ratios (Table 2). It indicated that there were variations among genotypes in rust disease and leaf morphology. Genotypes had a variation in leaf rust such as leaf-rust severity (5.2-25.84\%) (Table 3, Figure 3) of which G56 genotype derived from Dairi Regency performed the lowest leaf-rust severity $(5.21 \%)$. Genotypes showed also variation in leaf morphology. The results of this study were in line with Malau et al. (2018c); Malau \& Pandiangan (2018) who found variations in leaf morphology in genotypes that grew in North Sumatra. In contrast to leaf morphology, the ratio of leaf morphology (Table 4) such as the ratio of leaf length to leaf width, ratio of leaf length to leaf area, and the ratio of leaf width to leaf weight were not affected by the regency or sub regency or both (Table 2 ). This showed that these three ratios were not influenced by the growing environment. The expressivity stability of these ratios occurred because these ratios had high coefficients of heritability (Malau, 2019b).

Leaf-rust severity had highly significant negative genotypic correlation with leaf-rust incidence ( $r_{\mathrm{Gab}}=-$ $0.230^{*}$ ) (Table 5). Leaf-rust severity did not have significant genotypic correlation with branch-leaf rust. Leaf-rust incidence had significant negative correlation with branch-leaf rust $\left(r_{G a b}=-0.096^{*}\right)$.
Leaf-rust severity had highly significant positive genotypic and phenotypic correlations with ratios of leaf length and leaf area ( $\mathrm{r}_{\mathrm{Gab}}=0.254^{\star *}$ and $\mathrm{r}_{\mathrm{Pab}}=0.132^{\star *}$, respectively). Leaf-rust incidence did not correlate genotypically with any ratios of leaf morphology. Branch-leaf rust showed significant positive genotypic and phenotypic correlations with ratios of leaf length and leaf area.

In compared to leaf-rust incidence and branch-rust incidence, leaf-rust severity should be better used asmain selection criteria in coffee breeding for leaf-rust resistance because leaf-rust severity had the highest significant genotypic correlation $\left(r_{\mathrm{Gab}}=0.254^{\star *}\right)$. Let alone leaf-rust severity explains the level of damage caused by fungus Hemeleia vastatrix. The more the leaf-rust severity, the higher the level of damage. This fungus absorbs nutrients from the leaf on the underside of the leaf of the host plant (Ameson 2000). The more spores of this fungus germinate, the more nutrients will be absorbed by this fungus so the leaf becomes more nutrient deficiencies and then the leaf falls early. This leaf abscission significantly reduces the photosynthesis and then reduces production. Production of coffee fruit could be reduced up to $99 \%$ depending on the leaf-rust severity and the number of abscission leaves caused by this fungus. The results of this research supported Sera et al. (2010); Lima et al. (2018) who directly used rust-leaf severity to evaluate resistance of coffee plant in breeding process to search resistant genotype. The result of this research showed that rust-leaf incidence had no phenotypic correlation with rust-leaf severity ( $\mathrm{rPab}=-0,073 \mathrm{~ns})$ (Table 5). However, this result was in contrary with the results of research conducted by Silva-Acuña et al. (1999) who concluded that leaf-rust incidence could be used to estimate rust severity.

Ratio of leaf length to leaf area, however, might be used as an indirect selection criterion for leaf-rust severity because the ratio had significant positive genotypic correlation with leaf-rust severity ( $\mathrm{r}_{\mathrm{Gab}}=$ $\left.0.254^{\star *}\right)$ (Table 5). Regarding the correlation numbers,

Table 2 Analysis of variance and genetic components of leaf rust and leaf traits

\begin{tabular}{|c|c|c|c|c|c|c|c|c|c|c|}
\hline Parameter & $\begin{array}{l}\text { MS regency } \\
(p=7 ; d f=6)\end{array}$ & $\begin{array}{l}\text { MS subdistict } \\
(q=2 ; d f=7)\end{array}$ & $\begin{array}{l}\text { MS genotype } \\
(r=2 ; d f=14)\end{array}$ & $\begin{array}{c}\text { MS error (df }= \\
84)\end{array}$ & $\begin{array}{l}\text { F-ratio for } \\
\text { regency }\end{array}$ & $\begin{array}{c}\text { F-ratio for } \\
\text { sub } \\
\text { regency }\end{array}$ & $\begin{array}{l}\text { F-ratio for } \\
\text { genotype }\end{array}$ & $s 2 G$ & $s 2 E$ & $s 2 P$ \\
\hline \multicolumn{11}{|l|}{ Rust disease } \\
\hline LRS & 1970.66875 & 65.48090 & 63.94922 & 12.26887 & $30.10^{* *}$ & $1.02 \mathrm{~ns}$ & $5.21^{* *}$ & 5.168035 & 12.268868 & 17.436903 \\
\hline LRI & 10367.86316 & 346.74569 & 164.60851 & 9.49763 & $29.90^{* *}$ & $2.11 \mathrm{~ns}$ & $17.33^{* *}$ & 15.511088 & 9.497630 & 25.008718 \\
\hline BRI & 9250.94989 & 241.53762 & 199.94726 & 13.71423 & $38.30^{* *}$ & $1.21 \mathrm{~ns}$ & $14.58^{* *}$ & 18.623302 & 13.714233 & 32.337535 \\
\hline \multicolumn{11}{|l|}{ Leaf morphology } \\
\hline Leaf length (LL) & 208.02766 & 19.10252 & 12.20260 & 0.96886 & $10.89^{* *}$ & $1.57 \mathrm{~ns}$ & $12.59^{* *}$ & 1.123373 & 0.968864 & 2.092237 \\
\hline Leaf width (LWi) & 14.08990 & 9.75618 & 4.95895 & 0.18515 & $1.44 \mathrm{~ns}$ & $1.97 \mathrm{~ns}$ & $26.78^{* *}$ & 0.477379 & 0.185154 & 0.662533 \\
\hline Leaf weight (LWe) & 3.35942 & 0.04172 & 0.05307 & 0.01453 & $80.53^{* *}$ & $0.79 \mathrm{~ns}$ & $3.65^{* *}$ & 0.003854 & 0.014532 & 0.018386 \\
\hline Leaf area (LA) & 5009.23085 & 1222.43084 & 504.48282 & 23.57652 & $4.10^{\star}$ & $2.42^{*}$ & $21.40^{* *}$ & 48.090629 & 23.576524 & 71.667153 \\
\hline Ratio of LL/LWi & 5.95689 & 1.69598 & 2.00919 & 0.08481 & $3.51 \mathrm{~ns}$ & $0.84 \mathrm{~ns}$ & $23.69^{* *}$ & 0.192438 & 0.084813 & 0.277251 \\
\hline Ratio of LL/LWe & 43.05727 & 8.16200 & 7.12434 & 0.88252 & $5.28^{*}$ & $1.15 \mathrm{~ns}$ & $8.07^{* *}$ & 0.624182 & 0.882521 & 1.506703 \\
\hline Ratio of LL/LA & 0.05940 & 0.04128 & 0.02078 & 0.00083 & $1.44 \mathrm{~ns}$ & $1.99 \mathrm{~ns}$ & $24.95^{* *}$ & 0.001995 & 0.000833 & 0.002828 \\
\hline Ratio of LWi/LWe & 7.61022 & 3.59845 & 2.01103 & 0.13785 & $2.11 \mathrm{~ns}$ & $1.79 \mathrm{~ns}$ & $14.59^{* *}$ & 0.187317 & 0.137855 & 0.325172 \\
\hline Ratio of LWi/LA & 0.01697 & 0.00059 & 0.00193 & 0.00008 & $28.67^{* *}$ & $0.31 \mathrm{~ns}$ & $24.79^{* *}$ & 0.000186 & 0.000078 & 0.000264 \\
\hline Ratio of LWe/LA & 0.00071 & 0.00085 & 0.00032 & 0.00002 & $0.84 \mathrm{~ns}$ & $2.67^{*}$ & $13.52^{* *}$ & 0.000029 & 0.000024 & 0.000053 \\
\hline
\end{tabular}

Description: $\quad \mathrm{MS}=$ mean square $\mathrm{df}=$ degree of freedom, $\mathrm{LRS}=$ leaf-rust severity, $\mathrm{LRI}=$ leaf-rust incidence, $\mathrm{RBI}=$ branch-rust incidence, $\mathrm{LL}=$ leaf length $\mathrm{LWi}=$ leaf width, $\mathrm{LWe}=$ leaf weight, $\mathrm{LA}=$ leaf area, $\mathrm{F}$-table for regency at $\alpha 0.05=3.87$ and $\alpha 0.01=7.19, \mathrm{~F}$-table for sub regency at $\alpha 0.05=$ 2.17 and $\alpha 0.01=2.91$. F-table for genotype at $\alpha 0.05=1.30$ and $\alpha 0.01=1.44 . s^{2} G=$ estimated variance of genotype, $s^{2} E=$ estimated variance of error (mean square of error), and $s^{2} p=$ estimated variance of phenotype. 
Table 3 Leaf-rust disease and leaf morphology of 84 Arabica coffee genotypes

\begin{tabular}{|c|c|c|c|c|c|c|c|c|c|c|c|c|c|c|}
\hline Regency & Genotype & $\begin{array}{l}\text { LRS } \\
(\%)\end{array}$ & $\begin{array}{l}\text { LRI } \\
\text { (\%) }\end{array}$ & $\begin{array}{l}\text { BRI } \\
\text { (\%) }\end{array}$ & $\begin{array}{l}\text { LL } \\
(\mathrm{cm})\end{array}$ & $\begin{array}{l}\text { LWi } \\
(\mathrm{cm})\end{array}$ & $\begin{array}{c}\text { LWe } \\
\text { (g) }\end{array}$ & $\begin{array}{c}\mathrm{LA} \\
\left(\mathrm{cm}^{2}\right)\end{array}$ & $\begin{array}{l}\mathrm{LL} / \\
\mathrm{LWi}\end{array}$ & $\begin{array}{l}\mathrm{LL/} \\
\mathrm{LWe}\end{array}$ & $\begin{array}{l}\text { LL/ } \\
\text { LA }\end{array}$ & $\begin{array}{l}\mathrm{LWi} / \\
\mathrm{LWe}\end{array}$ & $\begin{array}{c}\text { LWi/ } \\
\text { LA }\end{array}$ & $\begin{array}{c}\mathrm{LWe} / \\
\mathrm{LA}\end{array}$ \\
\hline Humbang & G1 & 5.90 & 46.38 & 48.94 & 16.42 & 6.62 & 1.81 & 63.65 & 2.48 & 9.07 & 0.258 & 3.66 & 0.106 & 0.028 \\
\hline \multirow[t]{11}{*}{ Hasundutan } & G2 & 6.39 & 46.84 & 35.91 & 15.49 & 6.63 & 1.82 & 60.38 & 2.33 & 8.51 & 0.257 & 3.65 & 0.113 & 0.030 \\
\hline & G3 & 5.99 & 24.69 & 39.06 & 16.24 & 4.58 & 1.82 & 43.26 & 3.55 & 8.91 & 0.377 & 2.52 & 0.106 & 0.042 \\
\hline & G4 & 6.41 & 47.21 & 40.93 & 15.98 & 6.43 & 1.81 & 60.53 & 2.49 & 8.82 & 0.264 & 3.55 & 0.106 & 0.030 \\
\hline & G5 & 6.03 & 45.16 & 38.20 & 17.06 & 6.39 & 1.73 & 63.92 & 2.68 & 9.91 & 0.268 & 3.72 & 0.105 & 0.027 \\
\hline & G6 & 6.16 & 44.84 & 42.72 & 17.34 & 6.38 & 1.82 & 64.86 & 2.73 & 9.58 & 0.269 & 3.52 & 0.098 & 0.028 \\
\hline & G7 & 5.78 & 50.00 & 36.47 & 17.38 & 4.61 & 1.77 & 46.22 & 3.78 & 9.88 & 0.377 & 2.62 & 0.100 & 0.038 \\
\hline & G8 & 6.14 & 45.85 & 37.01 & 17.34 & 4.56 & 1.78 & 45.66 & 3.82 & 9.76 & 0.381 & 2.57 & 0.100 & 0.039 \\
\hline & G9 & 6.74 & 44.78 & 39.53 & 17.35 & 6.58 & 1.78 & 67.08 & 2.64 & 9.76 & 0.259 & 3.70 & 0.098 & 0.027 \\
\hline & G10 & 6.75 & 49.57 & 32.58 & 14.38 & 6.46 & 1.79 & 54.44 & 2.24 & 8.06 & 0.265 & 3.63 & 0.101 & 0.033 \\
\hline & G11 & 6.64 & 46.96 & 38.23 & 16.53 & 6.59 & 1.80 & 63.93 & 2.51 & 9.21 & 0.259 & 3.67 & 0.127 & 0.028 \\
\hline & G12 & 5.78 & 44.14 & 37.83 & 16.53 & 6.54 & 1.63 & 63.44 & 2.54 & 10.19 & 0.261 & 4.03 & 0.140 & 0.026 \\
\hline \multirow[t]{12}{*}{ Simalungun } & G13 & 9.24 & 65.50 & 54.54 & 13.90 & 4.65 & 1.81 & 39.35 & 2.99 & 7.67 & 0.368 & 2.57 & 0.129 & 0.048 \\
\hline & G14 & 9.18 & 65.85 & 54.29 & 16.53 & 4.54 & 1.81 & 59.93 & 3.65 & 8.79 & 0.383 & 2.41 & 0.130 & 0.044 \\
\hline & G15 & 9.57 & 56.24 & 55.18 & 16.40 & 4.54 & 1.83 & 42.89 & 3.62 & 8.98 & 0.383 & 2.49 & 0.072 & 0.043 \\
\hline & G16 & 9.91 & 66.38 & 44.58 & 16.43 & 6.48 & 1.81 & 54.61 & 2.54 & 9.11 & 0.263 & 3.59 & 0.104 & 0.029 \\
\hline & G17 & 9.66 & 62.72 & 45.98 & 15.12 & 4.54 & 1.83 & 39.58 & 3.34 & 8.28 & 0.383 & 2.48 & 0.081 & 0.047 \\
\hline & G18 & 9.88 & 61.99 & 49.33 & 16.53 & 6.28 & 1.81 & 60.84 & 2.64 & 9.16 & 0.273 & 3.47 & 0.103 & 0.030 \\
\hline & G19 & 10.91 & 61.78 & 55.42 & 14.61 & 4.54 & 1.78 & 37.64 & 3.23 & 8.22 & 0.391 & 2.55 & 0.082 & 0.048 \\
\hline & G20 & 10.51 & 65.51 & 30.20 & 14.90 & 5.91 & 1.78 & 51.42 & 2.53 & 8.39 & 0.291 & 3.33 & 0.115 & 0.035 \\
\hline & G21 & 9.19 & 59.85 & 54.37 & 15.25 & 6.68 & 1.77 & 60.80 & 2.29 & 8.64 & 0.252 & 3.78 & 0.110 & 0.029 \\
\hline & G22 & 10.18 & 63.93 & 53.52 & 14.97 & 4.36 & 1.77 & 36.87 & 3.45 & 8.49 & 0.410 & 2.47 & 0.119 & 0.049 \\
\hline & G23 & 9.24 & 55.69 & 52.48 & 14.83 & 6.51 & 1.82 & 56.16 & 2.28 & 8.16 & 0.264 & 3.58 & 0.116 & 0.033 \\
\hline & G24 & 10.17 & 63.98 & 58.20 & 14.89 & 6.46 & 1.85 & 56.30 & 2.31 & 8.06 & 0.265 & 3.49 & 0.115 & 0.033 \\
\hline \multirow[t]{12}{*}{ Pakpak Bharat } & G25 & 11.93 & 50.83 & 57.31 & 14.59 & 4.37 & 1.57 & 36.48 & 3.36 & 9.42 & 0.404 & 2.82 & 0.120 & 0.043 \\
\hline & G26 & 12.48 & 53.53 & 58.34 & 15.34 & 5.21 & 1.73 & 46.16 & 2.99 & 8.94 & 0.338 & 3.02 & 0.113 & 0.038 \\
\hline & G27 & 11.33 & 49.92 & 64.18 & 13.90 & 5.41 & 1.56 & 44.44 & 2.58 & 8.99 & 0.316 & 3.51 & 0.122 & 0.035 \\
\hline & G28 & 12.79 & 50.68 & 66.08 & 14.38 & 5.74 & 1.74 & 48.08 & 2.53 & 8.28 & 0.301 & 3.30 & 0.119 & 0.037 \\
\hline & G29 & 12.98 & 55.14 & 59.97 & 14.83 & 5.08 & 1.66 & 43.87 & 2.97 & 9.05 & 0.345 & 3.09 & 0.116 & 0.039 \\
\hline & G30 & 12.79 & 53.74 & 66.04 & 14.84 & 5.64 & 1.59 & 48.99 & 2.65 & 9.52 & 0.304 & 3.63 & 0.115 & 0.033 \\
\hline & G31 & 12.99 & 46.23 & 65.30 & 14.78 & 5.44 & 1.60 & 46.95 & 2.73 & 9.31 & 0.317 & 3.43 & 0.116 & 0.034 \\
\hline & G32 & 12.22 & 50.13 & 60.56 & 14.78 & 5.47 & 1.57 & 47.07 & 2.72 & 9.53 & 0.316 & 3.54 & 0.116 & 0.034 \\
\hline & G33 & 12.76 & 49.58 & 60.79 & 14.59 & 5.70 & 1.64 & 48.50 & 2.57 & 9.00 & 0.303 & 3.53 & 0.118 & 0.034 \\
\hline & G34 & 12.39 & 48.01 & 59.67 & 14.30 & 5.63 & 1.68 & 47.01 & 2.55 & 8.67 & 0.306 & 3.39 & 0.120 & 0.036 \\
\hline & G35 & 12.91 & 46.58 & 60.89 & 14.58 & 5.46 & 1.63 & 45.99 & 2.69 & 9.05 & 0.319 & 3.40 & 0.119 & 0.036 \\
\hline & G36 & 12.44 & 48.04 & 64.43 & 14.41 & 5.58 & 1.55 & 46.86 & 2.60 & 9.49 & 0.310 & 3.68 & 0.119 & 0.033 \\
\hline \multirow[t]{12}{*}{ Samosir } & G37 & 12.03 & 36.90 & 55.46 & 15.63 & 5.62 & 1.75 & 51.23 & 2.81 & 8.95 & 0.308 & 3.23 & 0.110 & 0.035 \\
\hline & G38 & 11.97 & 43.92 & 47.41 & 14.08 & 5.73 & 1.72 & 47.57 & 2.47 & 8.22 & 0.300 & 3.35 & 0.121 & 0.037 \\
\hline & G39 & 10.29 & 37.42 & 45.97 & 14.76 & 5.95 & 1.59 & 50.97 & 2.50 & 9.42 & 0.292 & 3.80 & 0.117 & 0.031 \\
\hline & G40 & 10.78 & 43.09 & 44.67 & 13.26 & 5.59 & 1.77 & 43.99 & 2.40 & 7.58 & 0.307 & 3.17 & 0.130 & 0.042 \\
\hline & G41 & 12.64 & 43.88 & 45.68 & 14.48 & 5.89 & 1.77 & 49.70 & 2.49 & 8.22 & 0.295 & 3.34 & 0.119 & 0.036 \\
\hline & G42 & 11.72 & 44.98 & 46.02 & 15.15 & 5.89 & 1.78 & 52.15 & 2.60 & 8.55 & 0.294 & 3.32 & 0.113 & 0.035 \\
\hline & G43 & 11.64 & 44.95 & 51.20 & 14.25 & 5.64 & 1.77 & 46.56 & 2.55 & 8.10 & 0.309 & 3.20 & 0.121 & 0.038 \\
\hline & G44 & 11.89 & 45.60 & 50.24 & 14.23 & 5.92 & 1.76 & 49.14 & 2.44 & 8.14 & 0.293 & 3.39 & 0.121 & 0.037 \\
\hline & G45 & 11.44 & 45.05 & 53.89 & 14.81 & 5.69 & 1.78 & 48.96 & 2.63 & 8.37 & 0.306 & 3.20 & 0.116 & 0.037 \\
\hline & G46 & 10.32 & 45.10 & 49.58 & 14.55 & 5.91 & 1.77 & 50.16 & 2.50 & 8.23 & 0.294 & 3.34 & 0.118 & 0.036 \\
\hline & G47 & 11.47 & 44.92 & 53.81 & 14.48 & 5.73 & 1.70 & 48.69 & 2.56 & 8.64 & 0.301 & 3.40 & 0.118 & 0.035 \\
\hline & G48 & 11.82 & 45.35 & 46.19 & 14.59 & 5.68 & 1.79 & 48.35 & 2.60 & 8.16 & 0.306 & 3.18 & 0.118 & 0.038 \\
\hline \multirow[t]{12}{*}{ Dairi } & G49 & 7.54 & 65.80 & 55.15 & 11.27 & 4.44 & 1.45 & 28.85 & 2.55 & 7.80 & 0.393 & 3.07 & 0.090 & 0.050 \\
\hline & G50 & 7.12 & 64.81 & 50.92 & 11.51 & 4.43 & 1.45 & 29.39 & 2.61 & 7.96 & 0.394 & 3.06 & 0.151 & 0.050 \\
\hline & G51 & 8.17 & 60.41 & 44.37 & 12.33 & 5.60 & 1.28 & 40.64 & 2.24 & 9.84 & 0.309 & 4.44 & 0.138 & 0.032 \\
\hline & G52 & 8.78 & 64.18 & 54.80 & 12.66 & 4.33 & 1.29 & 31.80 & 2.94 & 9.99 & 0.400 & 3.42 & 0.137 & 0.041 \\
\hline & G53 & 8.09 & 66.21 & 54.32 & 15.25 & 6.47 & 1.48 & 57.86 & 2.36 & 10.34 & 0.264 & 4.39 & 0.112 & 0.026 \\
\hline & G54 & 10.27 & 66.18 & 54.85 & 15.77 & 4.41 & 1.43 & 39.97 & 3.59 & 11.15 & 0.395 & 3.11 & 0.110 & 0.036 \\
\hline & G55 & 7.35 & 63.33 & 45.40 & 12.63 & 6.53 & 1.44 & 48.96 & 1.94 & 8.82 & 0.259 & 4.55 & 0.135 & 0.030 \\
\hline & G56 & 5.21 & 60.30 & 55.90 & 13.80 & 6.37 & 1.51 & 52.30 & 2.17 & 9.18 & 0.265 & 4.25 & 0.123 & 0.029 \\
\hline & G57 & 7.32 & 51.70 & 52.48 & 11.38 & 6.51 & 1.43 & 43.60 & 1.75 & 7.96 & 0.261 & 4.56 & 0.150 & 0.033 \\
\hline & G58 & 8.17 & 62.08 & 44.59 & 12.12 & 6.06 & 1.48 & 43.49 & 2.01 & 8.20 & 0.281 & 4.10 & 0.141 & 0.035 \\
\hline & G59 & 10.31 & 61.47 & 55.14 & 15.16 & 4.46 & 1.48 & 38.83 & 3.42 & 10.32 & 0.392 & 3.04 & 0.115 & 0.039 \\
\hline & G60 & 8.32 & 59.81 & 55.78 & 16.62 & 4.37 & 1.34 & 41.93 & 3.81 & 12.51 & 0.398 & 3.29 & 0.104 & 0.032 \\
\hline Tapanuli Utara & G61 & 11.68 & 42.18 & 45.65 & 12.60 & 4.53 & 1.40 & 32.85 & 2.80 & 9.03 & 0.385 & 3.25 & 0.138 & 0.043 \\
\hline & G62 & 13.84 & 44.00 & 44.76 & 12.10 & 5.63 & 1.47 & 39.65 & 2.16 & 8.26 & 0.305 & 3.84 & 0.144 & 0.038 \\
\hline & G63 & 13.21 & 41.26 & 46.14 & 12.39 & 5.84 & 1.38 & 42.72 & 2.14 & 9.07 & 0.294 & 4.24 & 0.138 & 0.033 \\
\hline & G64 & 14.91 & 43.85 & 45.57 & 12.60 & 5.15 & 1.46 & 37.71 & 2.48 & 8.68 & 0.340 & 3.54 & 0.137 & 0.039 \\
\hline & G65 & 14.11 & 39.16 & 45.12 & 12.81 & 5.58 & 1.41 & 41.46 & 2.34 & 9.12 & 0.315 & 3.95 & 0.135 & 0.035 \\
\hline & G66 & 12.00 & 42.22 & 46.07 & 13.33 & 5.47 & 1.46 & 42.44 & 2.50 & 9.19 & 0.322 & 3.78 & 0.129 & 0.035 \\
\hline & G67 & 11.92 & 37.53 & 53.37 & 13.81 & 5.60 & 1.50 & 45.13 & 2.47 & 9.24 & 0.306 & 3.75 & 0.124 & 0.033 \\
\hline & G68 & 13.38 & 35.68 & 42.55 & 12.22 & 5.63 & 1.49 & 12.17 & 2.18 & 8.27 & 0.305 & 3.81 & 0.140 & 0.037 \\
\hline & G69 & 12.12 & 42.11 & 44.10 & 12.11 & 5.71 & 1.48 & 40.29 & 2.13 & 8.20 & 0.301 & 3.88 & 0.144 & 0.037 \\
\hline & G70 & 25.84 & 34.34 & 55.70 & 11.41 & 4.63 & 1.48 & 30.45 & 2.47 & 7.71 & 0.377 & 3.12 & 0.152 & 0.049 \\
\hline & G71 & 12.30 & 42.99 & 54.92 & 13.09 & 4.73 & 1.44 & 35.63 & 2.78 & 9.12 & 0.367 & 3.29 & 0.134 & 0.041 \\
\hline & G72 & 14.41 & 44.56 & 54.66 & 10.55 & 5.74 & 1.43 & 35.02 & 1.88 & 7.42 & 0.307 & 4.01 & 0.163 & 0.041 \\
\hline
\end{tabular}


Table 3 Leaf-rust disease and leaf morphology of 84 Arabica coffee genotypes (advanced)

\begin{tabular}{|c|c|c|c|c|c|c|c|c|c|c|c|c|c|c|}
\hline \multirow{2}{*}{ Regency } & \multirow{2}{*}{ Genotype } & LRS & LRI & BRI & LL & LWi & LWe & LA & LL/ & LL/ & LL/ & LWi/ & LWi/ & LWe/ \\
\hline & & $(\%)$ & $(\%)$ & $(\%)$ & $(\mathrm{cm})$ & $(\mathrm{cm})$ & (g) & $\left(\mathrm{cm}^{2}\right)$ & LWi & LWe & LA & LWe & LA & LA \\
\hline \multirow[t]{17}{*}{ Toba Samosir } & G73 & 13.72 & 36.20 & 32.91 & 15.29 & 6.27 & 1.85 & 55.94 & 2.44 & 8.27 & 0.274 & 3.39 & 0.116 & 0.034 \\
\hline & G74 & 12.15 & 42.55 & 35.03 & 17.00 & 6.18 & 1.41 & 61.39 & 2.75 & 12.09 & 0.277 & 4.39 & 0.102 & 0.023 \\
\hline & G75 & 13.43 & 44.94 & 35.95 & 14.78 & 6.26 & 1.40 & 53.87 & 2.36 & 10.58 & 0.275 & 4.47 & 0.116 & 0.026 \\
\hline & G76 & 13.55 & 46.80 & 35.55 & 16.07 & 4.46 & 1.41 & 41.40 & 3.62 & 11.43 & 0.392 & 3.18 & 0.109 & 0.035 \\
\hline & G77 & 23.49 & 31.55 & 35.42 & 16.45 & 5.34 & 1.45 & 51.13 & 3.09 & 11.39 & 0.322 & 3.70 & 0.130 & 0.028 \\
\hline & G78 & 23.93 & 48.65 & 35.37 & 13.76 & 4.52 & 1.41 & 35.34 & 3.06 & 9.73 & 0.392 & 3.20 & 0.088 & 0.041 \\
\hline & G79 & 13.34 & 48.13 & 35.51 & 14.43 & 4.42 & 1.44 & 37.36 & 3.28 & 10.02 & 0.389 & 3.08 & 0.123 & 0.040 \\
\hline & G80 & 16.36 & 45.77 & 35.29 & 14.67 & 4.47 & 1.49 & 37.71 & 3.30 & 9.86 & 0.391 & 3.02 & 0.121 & 0.040 \\
\hline & G81 & 24.48 & 38.55 & 36.14 & 14.63 & 4.39 & 1.44 & 36.23 & 3.34 & 10.18 & 0.405 & 3.06 & 0.122 & 0.040 \\
\hline & G82 & 18.52 & 40.26 & 35.16 & 10.96 & 4.39 & 1.40 & 27.43 & 2.52 & 7.88 & 0.400 & 3.14 & 0.109 & 0.052 \\
\hline & G83 & 24.63 & 41.15 & 35.67 & 12.85 & 4.44 & 1.40 & 32.98 & 2.91 & 9.23 & 0.393 & 3.18 & 0.092 & 0.043 \\
\hline & G84 & 23.52 & 41.26 & 35.59 & 15.14 & 4.40 & 1.61 & 38.50 & 3.45 & 9.51 & 0.396 & 2.77 & 0.080 & 0.043 \\
\hline & Minimum & 5.21 & 24.39 & 30.20 & 10.55 & 4.33 & 1.28 & 12.17 & 1.75 & 7.42 & 0.252 & 2.41 & 0.072 & 0.023 \\
\hline & Maximum & 25.84 & 66.38 & 66.06 & 17.38 & 6.68 & 1.85 & 67.08 & 3.82 & 12.51 & 0.410 & 4.56 & 0.163 & 0.052 \\
\hline & Median & 11.44 & 46.58 & 46.19 & 14.63 & 5.63 & 1.60 & 46.16 & 2.57 & 8. 99 & 0.307 & 3.39 & 0.118 & 0.036 \\
\hline & Mean & 11.52 & 49.42 & 48.04 & 14.49 & 5.47 & 1.62 & 46.33 & 2.71 & 9.06 & 0.306 & 3.42 & 0.118 & 0.036 \\
\hline & sd & 0.64 & 0.56 & 0.68 & 0.18 & 0.08 & 0.02 & 0.89 & 0.05 & 0.17 & 0.005 & 0.07 & 0.002 & 0.001 \\
\hline
\end{tabular}

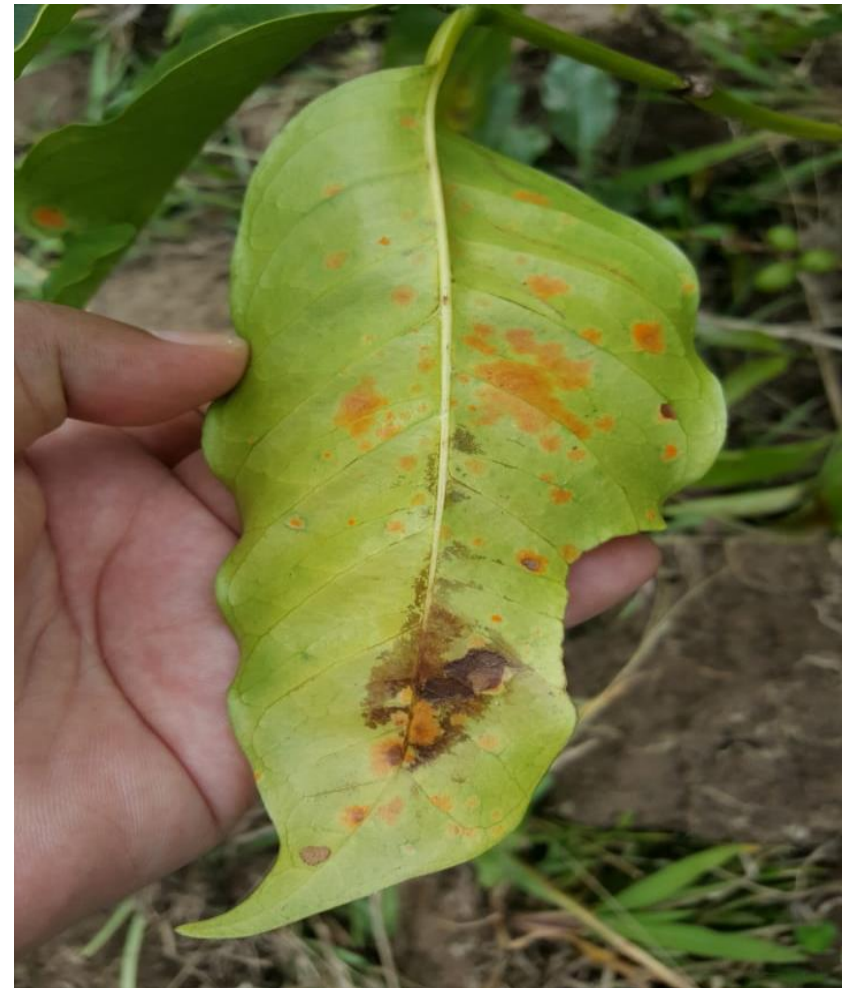

Figure 3 Leaf surface rusted by fungi Hemileia vastatrix with $15 \%$ leaf-rust severity.

happened because the number of data units in this study was quite large (Gomez \& Gomez 1984). Furthermore, by squaring the correlation coefficient number, the coefficient of determination is obtained at $6.5 \%$. That is, as much as $6.5 \%$ of variation in leaf-rust severity is explained by variations in the ratio of leaf length to area, the rest (93.5\%) is explained by the other unknown factors. In this such condition, namely the large number of data units that causes a small coefficient of determination, the focus of attention should be directed primarily to the significance of the relationship between parameters. The highly significant positive genotypic association between the ratio of leaf length to leaf area and leaf-rust severity was the decrease in the ratio of leaf length to leaf area that caused both characters to decrease simultaneously. The less the ratio is, the less the severity. For selection criteria, the ratio of leaf length to leaf area is even better chosen than leaf-rust severity because the first is more able to guarantee success than the latter. This result is because this ratio will be the same in different environments (Table 2). Besides, the ratio of leaf length to leaf area had a high coefficient of heritability $\left(\mathrm{H}^{2} \mathrm{~b}=\right.$ $70.54 \%)$ while leaf-rust severity showed a low one $\left(\mathrm{H}^{2} \mathrm{~b}\right.$ $=29.64 \%$ ) (Malau 2019b). Also, because the ratio of leaf length to leaf area showed a higher genetic advance in percentage of mean (GAM $=23.95 \%)$ than leaf-rust severity (GAM $=22.16 \%$ ), selection using the ratio as selection criteria can then produce a genotype performing lower leaf-rust severity in compare to selection using leaf-rust severity as criteria.

\section{CONCLUSION}

The regencies and sub regencies showed significant difference in rust disease and some leaf morphology. Genotypes had high significant difference in all rust-disease parameters, leaf morphology and the ratio of leaf morphology. Genotypes showed variation in leaf-rust severity (5.21-25.84\%) of which genotype G56 had the lowest leaf-rust severity. Genotypes had also variation in leaf morphology. Leaf length to leaf width ratio, leaf length to leaf area ratio, and leaf width to leaf weight ratio were not affected by the regency or sub regency or both. Leaf-rust severity showed highly significant positive genotypic and phenotypic correlation with leaf-length and leaf-area ratio.

In coffee breeding for leaf-rust resistance, leaf-rust severity could be better used as selection criteria rather than leaf-rust incidence and branch-rust incidence. Ratio of leaf length to leaf area could also be used as indirect selection criteria because the ratio showed 
Table 4 Ratio of leaf morphology of 84 Arabica coffee genotypes

\begin{tabular}{|c|c|c|c|c|c|c|c|}
\hline Regency & Genotype & $\begin{array}{l}\text { LL/ } \\
\text { LWi }\end{array}$ & $\begin{array}{c}\text { LL/ } \\
\text { LWe }\end{array}$ & $\begin{array}{l}\text { LL/ } \\
\text { LA }\end{array}$ & $\begin{array}{l}\text { LWi/ } \\
\text { LWe }\end{array}$ & $\begin{array}{c}\text { LWi/ } \\
\text { LA }\end{array}$ & $\begin{array}{c}\text { LWe/ } \\
\text { LA }\end{array}$ \\
\hline Humbang & G1 & 2.48 & 9.07 & 0.258 & 3.66 & 0.106 & 0.028 \\
\hline \multirow[t]{11}{*}{ Hasundutan } & G2 & 2.33 & 8.51 & 0.257 & 3.65 & 0.113 & 0.030 \\
\hline & G3 & 3.55 & 8.91 & 0.377 & 2.52 & 0.106 & 0.042 \\
\hline & G4 & 2.49 & 8.82 & 0.264 & 3.55 & 0.106 & 0.030 \\
\hline & G5 & 2.68 & 9.91 & 0.268 & 3.72 & 0.105 & 0.027 \\
\hline & G6 & 2.73 & 9.58 & 0.269 & 3.52 & 0.098 & 0.028 \\
\hline & G7 & 3.78 & 9.88 & 0.377 & 2.62 & 0.100 & 0.038 \\
\hline & G8 & 3.82 & 9.76 & 0.381 & 2.57 & 0.100 & 0.039 \\
\hline & G9 & 2.64 & 9.76 & 0.259 & 3.70 & 0.098 & 0.027 \\
\hline & G10 & 2.24 & 8.06 & 0.265 & 3.63 & 0.101 & 0.033 \\
\hline & G11 & 2.51 & 9.21 & 0.259 & 3.67 & 0.127 & 0.028 \\
\hline & G12 & 2.54 & 10.19 & 0.261 & 4.03 & 0.140 & 0.026 \\
\hline \multirow[t]{12}{*}{ Simalungun } & G13 & 2.99 & 7.67 & 0.368 & 2.57 & 0.129 & 0.048 \\
\hline & G14 & 3.65 & 8.79 & 0.383 & 2.41 & 0.130 & 0.044 \\
\hline & G15 & 3.62 & 8.98 & 0.383 & 2.49 & 0.072 & 0.043 \\
\hline & G16 & 2.54 & 9.11 & 0.263 & 3.59 & 0.104 & 0.029 \\
\hline & G17 & 3.34 & 8.28 & 0.383 & 2.48 & 0.081 & 0.047 \\
\hline & G18 & 2.64 & 9.16 & 0.273 & 3.47 & 0.103 & 0.030 \\
\hline & G19 & 3.23 & 8.22 & 0.391 & 2.55 & 0.082 & 0.048 \\
\hline & G20 & 2.53 & 8.39 & 0.291 & 3.33 & 0.115 & 0.035 \\
\hline & G21 & 2.29 & 8.64 & 0.252 & 3.78 & 0.110 & 0.029 \\
\hline & G22 & 3.45 & 8.49 & 0.410 & 2.47 & 0.119 & 0.049 \\
\hline & G23 & 2.28 & 8.16 & 0.264 & 3.58 & 0.116 & 0.033 \\
\hline & G24 & 2.31 & 8.06 & 0.265 & 3.49 & 0.115 & 0.033 \\
\hline \multirow[t]{12}{*}{ Pakpak Bharat } & G25 & 3.36 & 9.42 & 0.404 & 2.82 & 0.120 & 0.043 \\
\hline & G26 & 2.99 & 8.94 & 0.338 & 3.02 & 0.113 & 0.038 \\
\hline & G27 & 2.58 & 8.99 & 0.316 & 3.51 & 0.122 & 0.035 \\
\hline & G28 & 2.53 & 8.28 & 0.301 & 3.30 & 0.119 & 0.037 \\
\hline & G29 & 2.97 & 9.05 & 0.345 & 3.09 & 0.116 & 0.039 \\
\hline & G30 & 2.65 & 9.52 & 0.304 & 3.63 & 0.115 & 0.033 \\
\hline & G31 & 2.73 & 9.31 & 0.317 & 3.43 & 0.116 & 0.034 \\
\hline & G32 & 2.72 & 9.53 & 0.316 & 3.54 & 0.116 & 0.034 \\
\hline & G33 & 2.57 & 9.00 & 0.303 & 3.53 & 0.118 & 0.034 \\
\hline & G34 & 2.55 & 8.67 & 0.306 & 3.39 & 0.120 & 0.036 \\
\hline & G35 & 2.69 & 9.05 & 0.319 & 3.40 & 0.119 & 0.036 \\
\hline & G36 & 2.60 & 9.49 & 0.310 & 3.68 & 0.119 & 0.033 \\
\hline \multirow[t]{13}{*}{ Samosir } & G37 & 2.81 & 8.95 & 0.308 & 3.23 & 0.110 & 0.035 \\
\hline & G38 & 2.47 & 8.22 & 0.300 & 3.35 & 0.121 & 0.037 \\
\hline & G39 & 2.50 & 9.42 & 0.292 & 3.80 & 0.117 & 0.031 \\
\hline & G40 & 2.40 & 7.58 & 0.307 & 3.17 & 0.130 & 0.042 \\
\hline & G41 & 2.49 & 8.22 & 0.295 & 3.34 & 0.119 & 0.036 \\
\hline & G41 & 2.49 & 8.22 & 0.295 & 3.34 & 0.119 & 0.036 \\
\hline & G42 & 2.60 & 8.55 & 0.294 & 3.32 & 0.113 & 0.035 \\
\hline & G43 & 2.55 & 8.10 & 0.309 & 3.20 & 0.121 & 0.038 \\
\hline & G44 & 2.44 & 8.14 & 0.293 & 3.39 & 0.121 & 0.037 \\
\hline & G45 & 2.63 & 8.37 & 0.306 & 3.20 & 0.116 & 0.037 \\
\hline & G46 & 2.50 & 8.23 & 0.294 & 3.34 & 0.118 & 0.036 \\
\hline & G47 & 2.56 & 8.64 & 0.301 & 3.40 & 0.118 & 0.035 \\
\hline & G48 & 2.60 & 8.16 & 0.306 & 3.18 & 0.118 & 0.038 \\
\hline \multirow[t]{12}{*}{ Dairi } & G49 & 2.55 & 7.80 & 0.393 & 3.07 & 0.090 & 0.050 \\
\hline & G50 & 2.61 & 7.96 & 0.394 & 3.06 & 0.151 & 0.050 \\
\hline & G51 & 2.24 & 9.84 & 0.309 & 4.44 & 0.138 & 0.032 \\
\hline & G52 & 2.94 & 9.99 & 0.400 & 3.42 & 0.137 & 0.041 \\
\hline & G53 & 2.36 & 10.34 & 0.264 & 4.39 & 0.112 & 0.026 \\
\hline & G54 & 3.59 & 11.15 & 0.395 & 3.11 & 0.110 & 0.036 \\
\hline & G55 & 1.94 & 8.82 & 0.259 & 4.55 & 0.135 & 0.030 \\
\hline & G56 & 2.17 & 9.18 & 0.265 & 4.25 & 0.123 & 0.029 \\
\hline & G57 & 1.75 & 7.96 & 0.261 & 4.56 & 0.150 & 0.033 \\
\hline & G58 & 2.01 & 8.20 & 0.281 & 4.10 & 0.141 & 0.035 \\
\hline & G59 & 3.42 & 10.32 & 0.392 & 3.04 & 0.115 & 0.039 \\
\hline & G60 & 3.81 & 12.51 & 0.398 & 3.29 & 0.104 & 0.032 \\
\hline
\end{tabular}


Table 4 Ratio of leaf morphology of 84 Arabica coffee genotypes

\begin{tabular}{|c|c|c|c|c|c|c|c|}
\hline Regency & Genotype & $\begin{array}{l}\text { LL/ } \\
\text { LWi }\end{array}$ & $\begin{array}{l}\text { LL/ } \\
\text { LWe }\end{array}$ & $\begin{array}{l}\text { LL/ } \\
\text { LA }\end{array}$ & $\begin{array}{l}\text { LWi/ } \\
\text { LWe }\end{array}$ & $\begin{array}{c}\text { LWi/ } \\
\text { LA }\end{array}$ & $\begin{array}{c}\text { LWe/ } \\
\text { LA }\end{array}$ \\
\hline \multirow[t]{12}{*}{ Tapanuli Utara } & G61 & 2.80 & 9.03 & 0.385 & 3.25 & 0.138 & 0.043 \\
\hline & G62 & 2.16 & 8.26 & 0.305 & 3.84 & 0.144 & 0.038 \\
\hline & G63 & 2.14 & 9.07 & 0.294 & 4.24 & 0.138 & 0.033 \\
\hline & G64 & 2.48 & 8.68 & 0.340 & 3.54 & 0.137 & 0.039 \\
\hline & G65 & 2.34 & 9.12 & 0.315 & 3.95 & 0.135 & 0.035 \\
\hline & G66 & 2.50 & 9.19 & 0.322 & 3.78 & 0.129 & 0.035 \\
\hline & G67 & 2.47 & 9.24 & 0.306 & 3.75 & 0.124 & 0.033 \\
\hline & G68 & 2.18 & 8.27 & 0.305 & 3.81 & 0.140 & 0.037 \\
\hline & G69 & 2.13 & 8.20 & 0.301 & 3.88 & 0.144 & 0.037 \\
\hline & G70 & 2.47 & 7.71 & 0.377 & 3.12 & 0.152 & 0.049 \\
\hline & G71 & 2.78 & 9.12 & 0.367 & 3.29 & 0.134 & 0.041 \\
\hline & G72 & 1.88 & 7.42 & 0.307 & 4.01 & 0.163 & 0.041 \\
\hline \multirow[t]{17}{*}{ Toba Samosir } & G73 & 2.44 & 8.27 & 0.274 & 3.39 & 0.116 & 0.034 \\
\hline & G74 & 2.75 & 12.09 & 0.277 & 4.39 & 0.102 & 0.023 \\
\hline & G75 & 2.36 & 10.58 & 0.275 & 4.47 & 0.116 & 0.026 \\
\hline & G76 & 3.62 & 11.43 & 0.392 & 3.18 & 0.109 & 0.035 \\
\hline & G77 & 3.09 & 11.39 & 0.322 & 3.70 & 0.130 & 0.028 \\
\hline & G78 & 3.06 & 9.73 & 0.392 & 3.20 & 0.088 & 0.041 \\
\hline & G79 & 3.28 & 10.02 & 0.389 & 3.08 & 0.123 & 0.040 \\
\hline & G80 & 3.30 & 9.86 & 0.391 & 3.02 & 0.121 & 0.040 \\
\hline & G81 & 3.34 & 10.18 & 0.405 & 3.06 & 0.122 & 0.040 \\
\hline & G82 & 2.52 & 7.88 & 0.400 & 3.14 & 0.109 & 0.052 \\
\hline & G83 & 2.91 & 9.23 & 0.393 & 3.18 & 0.092 & 0.043 \\
\hline & G84 & 3.45 & 9.51 & 0.396 & 2.77 & 0.080 & 0.043 \\
\hline & Minimum & 1.75 & 7.42 & 0.252 & 2.41 & 0.072 & 0.023 \\
\hline & Maximum & 3.82 & 12.51 & 0.410 & 4.56 & 0.163 & 0.052 \\
\hline & Median & 2.57 & 8.99 & 0.307 & 3.39 & 0.118 & 0.036 \\
\hline & Mean & 2.71 & 9.06 & 0.323 & 3.42 & 0.118 & 0.036 \\
\hline & sd & 0.49 & 1.08 & 0.077 & 0.72 & 0.009 & 0.011 \\
\hline
\end{tabular}

Description: $\quad \mathrm{G}=$ genotype, $\mathrm{LRS}=$ leaf-rust severity, $\mathrm{LRI}=$ leaf-rust incidence, $\mathrm{BRI}=$ branch-rustincidence, $\mathrm{LL}=$ leaflength, $\mathrm{LWi}=$ leaf width, $\mathrm{LWe}=$ leaf weight, $\mathrm{LA}=$ leaf area, and $\mathrm{Sd}=$ standard deviation.

Table $5 s_{G a b}^{2}, s_{E a b}^{2}, s_{P a}^{2}, \operatorname{cov}_{G a b}, \operatorname{cov}_{P a b}, r_{G a b}$ and $r_{P a b}$ between genotypes

\begin{tabular}{|c|c|c|c|c|c|c|c|c|c|c|c|c|c|c|}
\hline & & LRS & LRI & BRI & LL & LWi & LWe & LA & $\begin{array}{l}\text { Ratio } \\
\text { LL/LWi }\end{array}$ & $\begin{array}{l}\text { Ratio } \\
\text { 니나e }\end{array}$ & $\begin{array}{l}\text { Ratio } \\
\text { LL/LA } \\
\end{array}$ & $\begin{array}{c}\text { Ratio } \\
\text { LWilLWe } \\
\end{array}$ & $\begin{array}{l}\text { Ratio } \\
\text { LWilLA } \\
\end{array}$ & $\begin{array}{c}\text { Ratio } \\
\text { LWe/LA }\end{array}$ \\
\hline & & & 1 & 2 & 3 & 4 & 5 & 6 & 7 & 9 & 8 & 10 & 11 & 12 \\
\hline \multirow[t]{7}{*}{ LRS } & $s_{\text {Gab }}^{2}$ & $x$ & 16.5622 & 25.5096 & 5.9426 & 4.8968 & 5.1621 & 45.5043 & 5.6560 & 5.6041 & 5.2215 & 4.8582 & 5.1699 & 5.1741 \\
\hline & $s_{E a b}^{2}$ & $\mathrm{x}$ & 22.8399 & 29.1927 & 13.2752 & 12.2753 & 12.2790 & 34.3900 & 12.4342 & 13.2096 & 12.2756 & 12.3072 & 12.2681 & 12.2695 \\
\hline & $S^{2}{ }_{p a b}$ & $\mathrm{x}$ & 39.4021 & 54.7022 & 19.2178 & 17.1721 & 17.4411 & 79.8944 & 18.0902 & 18.8136 & 17.4972 & 17.1653 & 17.4380 & 17.4436 \\
\hline & $\operatorname{cov}_{\text {Gab }}$ & $\mathrm{x}$ & -2.0585 & 0.8591 & -0.1744 & -0.3743 & -0.0049 & -3.8772 & 0.1478 & -0.0941 & 0.0258 & -0.2486 & 0.0008 & 0.0030 \\
\hline & $\operatorname{CON}_{\mathrm{Patb}}$ & $\mathrm{x}$ & -1.5218 & 2.4639 & -0.1557 & -0.4637 & -0.0071 & -4.6048 & 0.1880 & -0.0650 & 0.0287 & -0.2984 & 0.0004 & 0.0033 \\
\hline & Tabo & $\mathrm{x}$ & $-0.230^{* *}$ & $0.088 \mathrm{~ns}$ & $-0.072 \mathrm{~ns}$ & $-0.238^{* *}$ & $-0.035 \mathrm{~ns}$ & $-0.246^{* *}$ & $0.148^{* *}$ & $-0.052 \mathrm{~ns}$ & $0.254^{* *}$ & $-0.253^{* *}$ & $0.027 \mathrm{~ns}$ & $0.243^{* *}$ \\
\hline & $I_{p a b}$ & $x$ & $-0.073 \mathrm{~ns}$ & $0.104^{*}$ & $-0.026 \mathrm{~ns}$ & $-0.136^{* *}$ & $-0.013 \mathrm{~ns}$ & $-0.130^{* *}$ & $0.086 \mathrm{~ns}$ & $-0.013 \mathrm{~ns}$ & $0.129^{* *}$ & $-0.125^{* *}$ & $0.006 \mathrm{~ns}$ & $0.109^{*}$ \\
\hline \multirow[t]{7}{*}{ LRI } & $s^{2}=$ & & $\mathrm{x}$ & 30.8614 & 16.4837 & 15.4912 & 15.4889 & 67.1009 & 15.4912 & 16.2157 & 15.4959 & 15.8758 & 15.5178 & 15.5088 \\
\hline & $s^{2}$ Eat & & $\mathrm{x}$ & 24.0966 & 10.1845 & 9.5517 & 9.4884 & 32.4458 & 9.5517 & 10.3601 & 9.4981 & 9.7066 & 9.4986 & 9.4978 \\
\hline & $s^{2}{ }_{P a b}$ & & $\mathrm{x}$ & 54.9579 & 26.6682 & 25.0429 & 24.9773 & 99.5467 & 25.0429 & 26.5758 & 24.9939 & 25.5824 & 25.0164 & 25.0066 \\
\hline & $\operatorname{cov}_{\mathrm{Gat}}$ & & $x$ & -1.6365 & -0.0754 & -0.2486 & -0.0130 & 1.7496 & -0.1062 & 0.0402 & -0.0086 & 0.0887 & 0.0033 & -0.0012 \\
\hline & $\operatorname{cov}_{\mathrm{Pab}}$ & & $\mathrm{x}$ & -1.1942 & -0.2164 & -0.3142 & -0.0249 & 1.4354 & -0.1215 & 0.0302 & -0.0088 & 0.1242 & 0.0037 & -0.0011 \\
\hline & $T_{G}$ & & $\mathrm{x}$ & $-0.096^{*}$ & $-0.018 \mathrm{~ns}$ & $-0.091^{*}$ & $-0.053 \mathrm{~ns}$ & $0.064 \mathrm{~ns}$ & $-0.061 \mathrm{~ns}$ & $0.013 \mathrm{~ns}$ & $-0.049 \mathrm{~ns}$ & $0.052 \mathrm{~ns}$ & $0.061 \mathrm{~ns}$ & $-0.055 n s$ \\
\hline & $\tau_{\mathrm{p}}$ & & $\mathrm{x}$ & $0.099^{*}$ & $-0.030 \mathrm{~ns}$ & $-0.077 \mathrm{~ns}$ & $-0.037 n s$ & $0.034 \mathrm{~ns}$ & $-0.046 \mathrm{~ns}$ & $0.005 \mathrm{~ns}$ & $-0.033 \mathrm{~ns}$ & $0.044 \mathrm{~ns}$ & $0.046 \mathrm{~ns}$ & $-0.030 \mathrm{~ns}$ \\
\hline \multirow[t]{7}{*}{ BRI } & $s_{G a b}^{2}$ & & & $\mathrm{x}$ & 21.6223 & 18.2200 & 18.6160 & 64.4995 & 19.6916 & 20.5315 & 18.6971 & 18.1669 & 18.6114 & 18.6268 \\
\hline & $s^{2}=a b$ & & & $\mathrm{x}$ & 14.8330 & 13.6848 & 13.7652 & 35.8281 & 13.9157 & 14.5138 & 13.7228 & 13.6641 & 13.7131 & 13.7160 \\
\hline & $s^{2}=$ & & & $\mathrm{x}$ & 36.4553 & 31.9047 & 32.3812 & 100.3277 & 33.6073 & 35.0453 & 32.4200 & 31.8310 & 32.3245 & 32.3427 \\
\hline & $\operatorname{cov}_{\mathrm{Gab}}$ & & & $x$ & 0.9378 & -0.4404 & -0.0056 & -1.1072 & 0.4380 & 0.6420 & 0.0359 & -0.3219 & -0.0060 & 0.0017 \\
\hline & $\operatorname{cov}_{P a b}$ & & & $\mathrm{x}$ & 1.0128 & -0.5477 & 0.0126 & -1.8385 & 0.4963 & 0.6005 & 0.0398 & -0.4159 & -0.0067 & 0.0026 \\
\hline & $r_{\text {cab }}$ & & & $x$ & $0.205^{* *}$ & $-0.148^{* *}$ & $-0.021 \mathrm{~ns}$ & $-0.037 \mathrm{~ns}$ & $0.231^{* *}$ & $0.188^{* *}$ & $0.186^{* *}$ & $-0.172^{* *}$ & $-0.103^{*}$ & $0.073 \mathrm{~ns}$ \\
\hline & $\Gamma_{\text {Pab }}$ & & & $x$ & $0.123^{* *}$ & $-0.118^{* *}$ & $0.016 \mathrm{~ns}$ & $-0.038 \mathrm{~ns}$ & $0.166^{* *}$ & $0.086 \mathrm{~ns}$ & $0.132^{* *}$ & $-0.128^{* *}$ & $-0.072 \mathrm{~ns}$ & $0.062 \mathrm{~ns}$ \\
\hline
\end{tabular}

Description: $n=84, \mathrm{LRS}=$ leaf-rust severity, $\mathrm{LRI}=$ leaf-rust incidence, $\mathrm{RBI}=$ branch-rust incidence, $\mathrm{LL}=$ leaf length, $\mathrm{LWi}=$ leaf width, $\mathrm{LWe}$ $=$ leaf weight, $L A=$ leaf area, $s^{2}$ Gab $=$ estimated variance of genotype of dummy phenotypes $(a+b), s^{2}$ Eab $=$ estimated variance of error (mean square of error) of dummy phenotypes $(a+b), s^{2}$ pab $=$ estimated variance of phenotype of dummy phenotypes $(a+b), \operatorname{cov}_{G a b}=$ genetic covariance between phenotypes $a$ and $b, \operatorname{cov}_{\mathrm{Pab}}=$ phenotypic covariance between phenotypes a and $\mathrm{b}, \mathrm{r}_{\mathrm{Gab}}=$ genotypic correlation between phenotype $\mathrm{a}$ and $\mathrm{b}, \mathrm{r}_{\mathrm{Pab}}=$ phenotypic correlation between phenotype $\mathrm{a}$ and $\mathrm{b}, \mathrm{r}$ table at $\alpha 0.05=0.088, r$ table at $\alpha 0.01=0.115, n s=$ not significant, ${ }^{*}=$ significance at $\alpha=0.05$, and ${ }^{*}=$ significance at $\alpha=0.01$.

significant genotypic correlation with leaf-rust severity $\left(r_{\mathrm{Gab}}=0.254^{\star *}\right)$. However, the ratio of leaf length to leaf area is even better chosen for selection criteria rather than leaf-rust severity because the ratio was not affected by the environments.

\section{ACKNOWLEDGEMENT}

The authors thank the Ministry of Research, Technology, and Higher Education for funding this research with grant number 0100/E5.1/PE/2015 dated January 19, 2015. 


\section{REFERENCES}

Andreazi E, Sera GH, Faria $R T$, de $T$, Sera $T$, Shigueoka LH, Carvalho FG, Carducci FC, Chamlet D. 2015. Performance of $f 1$ hybrids of arabica coffee with simultaneous resistance to leaf rust, bacterial blight and leaf miner. Coffee Science. 10(3): 375-382.

Ameson. 2000. Coffee rust. The Plant Health Instructor. [internet] [Accessed 8 February 2014]. Available on: https://www.apsnet.org/edcenter/intropp/lessons/fu ngi/basidiomycetes/pages/coffeerust.aspx. https:// doi.org/10.1094/PHI-I-2000-0718-02

Avelino A, Cristancho M, Georgiou S, Imbach $P$, Aguilar L, Bornemann G, Läderach P, Anzueto F, Hruska AJ, Morales C. 2015. The coffee rust crises in Colombia and Central America (2008-2013): impacts, plausible causes and proposed solutions. Food Security. 7(2): 303-321. https://doi.org/ 10.1007/s12571-015-0446-9

[BMKG] Badan Meteorologi, Klimatologi dan Geofosika. 2017. Weather forecast for North Sumatera. [internet] [Accessed 28 August 2017]. Available on: http://www.bmkg.go.id/cuaca/ prakiraan-cuaca-indonesia.bmkg?Prov=34\&Nama Prov $=$ Sumatera\%20Utara.

Boudrot A, Pico J, Merle I, Granados E, Vilchez S, Tixier P, Filho EDMV, Casanoves F, Tapia A, Allinne C, Rice RA, Avelino J. 2016. Shade Effects on the Dispersal of Airborne Hemileia vastatrix Uredospores. Phytopathology. 106(6): 572-580. https://doi.org/10.1094/PHYTO-02-15-0058-R

Caicedo BLC, Hernando A, Guerrero C, Roux J, Wingfield MJ. 2013. New coffee (Coffea arabica) genotypes derived from Coffea canephora exhibiting high levels of resistance to leaf rust and Ceratocystis canker. Tropical plant pathology. 38(6): 485-494. https://doi.org/10.1590/S198256762013000600004

Carvalho CR, Fernandes RC, Carvalho GMA, Barreto, RW, Evans HC. 2011. Cryptosexuality and the Genetic Diversity Paradox in Coffee Rust, Hemileia vastatrix. PLoS ONE. 6(11): e26387. https:// doi.org/10.1371/journal.pone.0026387

[DGEC] Directorate General of Estate Crops. 2017. Tree crop estate statistics of Indonesia 2016-2018, Cofee. Jakarta (ID).

Gomez AK, Gomez AA. 1984. Statistical procedures for agricultural research. New York (US):John Wiley \& Sons.

Harni R, Taufiq E, Martono B. 2015. Reistance of liberica coffee against leaf rust disease (Hemileia vastatrix B. et Br.) in kepulauan Meranti. Jurnal Tanaman Industri dan Penyegar. 2(1): 35-42. https://doi.org/10.21082/jtidp.v2n1.2015.p35-42
Hindorf H, Omondi CO. 2011. A review of three major fungal diseases of Coffea Arabica L. in the rainforests of Ethiopia and progress in breeding for resistance in Kenya. Journal Advance Research. 2: 109-120. https://doi.org/10.1016/j.jare.2010.08. 006

Jeffuka C, Fininsa C, Adugna G, Hindorf H. 2010. Coffee leaf rust epicemic (Hemileia vastatrix) in Montane coffee (Coffea Arabica L.) forests in southwestern Ethiopia. East African Journal of Sciences. 4(2): 86-95. https://doi.org/10.4314/ eajsci.v4i2.71530

Lima AED, Junior HGS, Castro EDM, Carvalho SPD, Silva FFD, Junior SDL, Carvalho AMD. 2019. Incidence and severity of coffee leaf rust, cercosporiosis and coffee leaf miner in coffee progenies. Coffee Science. 13(3): 349-355. https:// doi.org/10.25186/cs.v13i3.1459

López-Bravo DF, Virginio-Filho E, de M, Avelino J, 2012. Shade is conducive to coffee rust as compared to full sun exposure under standardized fruit load conditions. Crop Protection. 38: 21-29. https://doi.org/10.1016/j.cropro.2012.03.011

Malau S, Siagian A, Sirait B, Pandiangan, S. 2017. Performance of coffee origin and genotype in organoleptic and physical quality of Arabica coffee in North Sumatra Province of Indonesia. $1^{\text {st }}$ Nommensen International Conferennce on Technology and Engineering: Advancements in Technology and Engineering. IOP Conference Series: Materials Science and Engineering 237: 012035. https://doi.org/10.1088/1757-899X/237/1/ 012035

Malau S, Pandiangan S. 2018. Variation of Arabica Coffee (Coffea arabica L.) Growing in North Sumatra Province of Indonesia Based on Morphology. Jurnal Agronomi Indonesia. 46(3): 314-321. https://doi.org/10.24831/jai.v46i3.193 42

Malau S, Siagian A, Sirait B, Ambarita H. 2018a. Germination performance of Coffea arabica $\mathrm{L}$. genotypes from different altitude, precipitation and temperature of seeds producing farms in North Sumatra of Indonesia. International Conference on Agribusiness, Food and Agro-Technology. IOP Conference Series: Earth and Environmental Sience. 205: 012013. https://doi.org/10.1088/17551315/205/1/012013

Malau S, Siagian A, Sirait B, Ambarita H. 2018b. PlantgrowthofgenotypesofArabicacoffeeonwater stress. $2^{\text {nd }}$ Nommensen International Conference on Technology and Engineering 2018: Sustainable Engineering and Technology Innovation and Application. IOP Conference Series: Material Science and Engineering.420: 012066. https:// doi.org/10.1088/1757-899X/420/1/012066 
Malau S, Lumbanraja P, Pandiangan S, Tarigan JR, Tindaon F. 2018c. Performance of Coffea arabica $L$. in changing climate of North Sumatra of Indonesia. Scientia Agriculturae Bohemica. 49(4): 340-349. https://doi.org/10.2478/sab-2018-0041

Malau S, Siagian A, Sirait B, Ambarita $\mathrm{H}$, Pandiangan S, Sihotang MR, Naibaho B, Simanjuntak R. 2018d. Variability of organoleptic quality of Arabica coffee. Anadolu Tarım Bilimleri Dergisi. 33(3): 241-245. https://doi.org/10.7161/omuanajas.405418

Malau S, SiagianA, Sihotang MR, Simanjuntak $R$, Hutabarat, NDM R. 2019a.Saepo Modi and Correlation among Statistical Methods for Measuring of Phenotypic Stability. Jurnal Pertanian Tropik. 6(2): 238-249. https://doi.org/10.32734/ jpt.v6i2.3159

Malau S, Sihotang MR, Simanjuntak R. 2019b. Effect of genotype and storage duration on taste and ecovalence of roasted beans of Arabica coffee (Coffea arabicaL.). Tarumanagara International Conference on the Applications of Technology and Engineering. IOP Conference Series: Material Science and Engineering. 508: 012115.https://doi.org/10.1088/ 1757-899X/508/1/012115

Malau S. 2019a. Resistance variability of arabica coffee genotypes (Coffea arabica L.) to leaf rust disease (Hemileia vastatrix). Jurnal Tanaman Industri dan Penyegar. 6(2): 69-78. https://doi.org/10.21082/jtidp.v6n2.2019.p69-78

Malau, S. 2019b. Phenotypic and genotypic correlation among taste attributes and weight of green bean in Arabica coffee (Coffea Arabica L.). 2nd International Conference on Agriculture, Environment, and Food Security (AEFS) 2018. IOP Conference Series: Earth and Environmental Science.260: 012120. https://doi.org/10.1088/1755-1315/260/1/012120

Mayo O. 1987. The Theory of Plant Breeding. New York (US): Oxford University Press. 334p.

McCook S. 2006. Global rust belt: Hemileia vastatrix and the ecological integration of world coffee production since 1850. Journal of Global History 1(2): 177-195. https://doi.org/10.1017/S1740022 $80600012 X$

Quinn GP, Keough MJ. 2002. Experimental design and data analysis for biologists. Cambridge (EN):
Cambridge University Press. NY. 537p. https:// doi.org/10.1017/CBO9780511806384

Rodrigues MN, Ferrao RG, Fonseca AF. Mendonca RF, Martins LD, Tomaz MA. 2012. Crop yield of conilon coffee plants of different levels of vegetative vigor and rust severity. Nucleus. 9(2): 1-6. https:// doi.org/10.3738/1982.2278.780

Sera GH, Sera T, Fonseca IC, de B, Ito DS. 2010. Resistance to leaf rust in coffee cultivars. Coffee Science. 5(1): 59-66.

Silva-Acuña R, Maffia LA, Zambolim L, Berger RD. 1999. Incidence-Severity relationship in the pathosystem Coffea Arabica-Hemeleia vastatrix. Plant Disease. 83: 186-188. https://doi.org/ 10.1094/PDIS.1999.83.2.186

Siska RKW, Lubis L, Lisnawita. 2018. Serangan Karat Daun Kopi (Hemileia vastatrix $\mathrm{B}$ et $\mathrm{Br}$ ) pada Tanaman Kopi Arabika di Perkebunan Rakyat Kabupaten Mandailing Natal Sumatera Utara. ARN Cofference Series. 1: 82-86. https://doi.org/ 10.32734/anr.v1i1.101

Sudrajat A. 2009. Mapping the Oldeman SchmidthFergusson climate classifications as an effort to utilize climate resources in natural resource management in North Sumatra. [Thesis]. Medan (ID): North Sumatra University.

Talhinhas $P$, Batista D, Diniz I, Vieira A, Silva DN, Loureiro A, Tavares S, Pereira AP, Azinheira HG, Guerra-Guimara L, Varzea V, Silva M, do Z, Ceu. 2017. The Coffee Leaf Rust pathogen Hemileia vastatrix: One and a half centuries around the tropics: Coffee Leaf Rust caused by Hemileia vastatrix. Molecular Plant Pathology. 18(8): 1-13. https://doi.org/10.1111/mpp.12512

Unigarro-Muñoz CA, Hernández-Arredondo JD, Montoya-Restrepo EC, Medina-Rivera RD, IbarraRuales LN,Carmona-González CY, Flórez-Ramos CP. 2015. Estimation of leaf area in coffee leaves (Coffea arabica L.) of the Castillo variety. Bragantia, Campina. 74(4): 412-416.

Zeru A, Assefa F, Adugna G, Hindorf H. 2009. Occurrence of fungal diseases of Coffea arabica $\mathrm{L}$. in Montane rainforests of Ethiopia. Journal of Applied Botany and Food Quality. 2: 148-151. https://doi.org/10.1590/1678-4499.0026 